\title{
Diversité, usages vétérinaires et vulnérabilité des plantes médicinales au
} Nord-Bénin

\author{
H. G. DASSOU ${ }^{1 *}$, C. A. OGNI ${ }^{2}$, H. YEDOMONHAN ${ }^{1}$, A. C. ADOMOU ${ }^{1}$, \\ M. TOSSOU ${ }^{1}$, J. T. DOUGNON ${ }^{2}$ et A. AKOEGNINOU ${ }^{1}$ \\ ${ }^{1}$ Laboratoire de Botanique et Ecologie Végétale, Département de Biologie Végétale, Faculté des Sciences et \\ Techniques, Université d'Abomey-Calavi (UAC), 01 BP 4521 Cotonou, Benin. \\ ${ }^{2}$ Laboratoire de Recherches et de Biologie Appliquée, Université d'Abomey-Calavi, \\ 01 BP 2009 Cotonou, Benin. \\ *Auteur correspondant, E-mail : daspice2@gmail.com,Tél. : (+229) 95202552
}

\section{RESUME}

Au Bénin, les animaux domestiques continuent de payer un lourd tribut à diverses pathologies. Une enquête a été conduite au Nord-Bénin pour recenser les recettes à usages vétérinaires, sélectionner les plus crédibles et évaluer la vulnérabilité des plantes impliquées. Elle a consisté à des interviews individuelles semistructurées auprès de 60 éleveurs. Au total 56 espèces végétales ont été citées. Elles sont reparties en 50 genres et 22 familles. Les familles les plus représentées en espèces sont : Leguminosae (34\%) et Combretaceae (11\%). Au total 86 recettes ont été recensées pour traiter 31 pathologies avec la Fièvre aphteuse comme la plus fréquente (19\%). Les fréquences de recettes (Fr) et de citation des recettes (Fcr) ont permis de sélectionner celles qui sont plus crédibles. Les écorces de tige sont plus citées avec un pourcentage de $61 \%$. Huit (08) modes de préparation sont indiqués avec la décoction (49\%) comme la plus fréquente. Trente et un (31) espèces se sont révélées vulnérables. Cette étude a permis la sélection de quelques recettes crédibles. Les recherches phytochimiques ultérieures montreront les potentialités réelles des plantes citées dans le traitement des pathologies animales.

(C) 2014 International Formulae Group. All rights reserved.

Mots clés : Plantes, médecine vétérinaire, Fr, Fcr, Bénin.

\section{INTRODUCTION}

Le Bénin est subdivisé en huit (08) zones agroécologiques suivant la trilogie solclimat-culture auxquelles correspondent des systèmes de productions et des spéculations spécifiques.

$\mathrm{Au}$ niveau de ces 8 zones agroécologiques, la zone II ou la zone cotonnière du Nord-Bénin montre un taux relativement élevé en espèces animales. En effet, elle compte $33 \%$ du cheptel bovin béninois, $21 \%$ du cheptel d'ovins, $09 \%$ de caprins, $07 \%$ de volailles et $4 \%$ du cheptel de porcins (DE, 2010).

Malheureusement, les animaux élevés sont affectés par de nombreuses et graves maladies qui occasionnent de lourdes pertes économiques et protéiques pour les éleveurs en particulier et la population béninoise en général. Au nombre de ces maladies figurent 
la peste porcine avec $100 \%$ de mortalité des porcs élevés. Elle est suivie de la maladie de Newcastle et de la Peste de Petits Ruminants (PPR) qui déciment respectivement $36 \%$ et $18 \%$ des animaux affectés (DE, 2010).

La prise en charge est souvent difficile pour les éleveurs car la diffusion des produits vétérinaires est très limitée, leur coût est élevé, leur efficacité et leur utilisation restent peu connues. Cependant, les éleveurs vivant avec leurs animaux et ayant toujours observé leurs comportements, ont pu asseoir jour après jour l'ethnomédecine vétérinaire (Upadhyay et al., 2011). Selon ces auteurs, les connaissances liées aux recettes dans le domaine de la pharmacopée traditionnelle sont transmises oralement de génération en génération au sein de la société et demeurent un patrimoine soit de la famille, soit d'un groupe social particulier du village ou de la contrée. Par conséquent le risque de disparition de ces savoirs endogènes est très grand s'ils ne sont pas documentés (Devendrakumar et Anbazhagan, 2012).

C'est dans ce cadre que s'inscrit le présent travail dont l'objectif global est de documenter les savoirs endogènes des populations en matière de la médecine ethnovétérinaire en vue de sa valorisation au Bénin. De façon spécifique, il s'agit de :

- inventorier les plantes utilisées et documenter les recettes associées dans le traitement des pathologies animales dans la zone cotonnière du Nord-Bénin ;

- apprécier le consensus sur l'utilisation des plantes médicinales dans le traitement des catégories de maladies et sélectionner les recettes crédibles ;

- évaluer la vulnérabilité des espèces végétales en pharmacopée vétérinaire dans un contexte d'utilisation durable.

\section{MATERIEL ET METHODES}

\section{Site de l'étude}

L'étude a été conduite dans la zone agroécologique II, encore appelée zone cotonnière du Bénin. D’une superficie de $20.930 \mathrm{~km}^{2}$, elle est comprise entre les parallèles $10^{\circ} 29^{\prime} 34^{\prime \prime}$ et $11^{\circ} 45^{\prime} 25^{\prime \prime}$ de latitude
Nord et les méridiens $01^{\circ} 42^{\prime} 54^{\prime \prime}$ et $3^{\circ} 43^{\prime} 51^{\prime \prime}$ de longitude Est (Figure 1). Elle comprend les communes de Kérou, Ségbana, Banikoara, Kandi et Gogounou (MEPN, 2008).

Le climat est de type tropical sec caractérisé par deux saisons: une saison pluvieuse de Mars à Septembre et une saison sèche d'Octobre à Février (Akoègninou, 2004).

Sur le plan phytogéographique, le milieu d'étude appartient au district Borgounord dont la flore est riche de 320 espèces végétales (Adomou, 2011). Selon le même auteur, la végétation est une mosaïque de forêts denses sèches à Anogeissus leiocarpa, de forêts claires à Isoberlinia, de savanes boisées à Monotes kerstingii, de savanes arborées et arbustives à Burkea africana et à Combretum. On y rencontre aussi des savanes herbeuses à Panicum et à Echinochloa, des forêts galeries dominées par les espèces telles que: Berlinia grandiflora, Cola laurifolia, Syzygium guineense, etc.

Considérant la démographie, la population de la zone est de 731.680 habitants et les groupes ethniques présents sont les Bariba (38\%), Peulh (22\%), Dendi (21\%), Mokolé (12\%), Gando (7\%) (INSAE, 2013). Selon FAO (2010), parmi les principales activités figure l'agriculture employant $42 \%$ de la population qui font la culture du sorgho, du maïs, du riz et surtout du coton. Elle est suivie de l'élevage qui emploie $33 \%$ de la population. Il faut noter que les bovins constituent les animaux les plus élevés avec un taux de $33 \%$ de l'effectif national. On y compte aussi les volailles avec un taux de $6 \%$ de l'effectif national (DE, 2010).

\section{Méthode \\ Enquête ethnovétérinaire}

Les villages qui montrent une forte concentration en animaux ont été indiqués par les Responsables du Développement Rural (RDR) et ont fait l'objet d'investigation. Au total, 60 éleveurs ont été enquêtés dans les communes de Gogounou, de Kandi et de Banikoara à raison de 20 personnes par commune. Ils ont été identifiés par les 
Conseillés en Production Animale (CPA) des Secteurs Communaux pour le Développement Agricole (SCDA) sur la base de leur expérience en médecine ethnovétérinaire. Un Doctorant vétérinaire de l'Ecole Polytechnique de l'Université d'AbomeyCalavi (EPAC) a été associé pour faire la typologie des maladies sur la base des symptômes spécifiques et généraux des pathologies.

Des enquêtes ont été réalisées par des interviews individuelles à l'aide d'un interprète. Les différentes rubriques du questionnaire sont: le profil de l'enquêté (âge, sexe, niveau d'instruction, taille du troupeau), les noms locaux et les organes végétaux utilisés pour les plantes, les recettes relatives aux plantes et les maladies traitées, l'origine de la connaissance ethnovétérinaire (famille, amis éleveurs ou institution comme le SCDA).

Les plantes citées par les enquêtés ont été aussitôt récoltées et échantillonnées. L'identification taxonomique a été réalisée à l'Herbier National du Bénin par comparaison avec les herbiers de référence ou avec la Flore analytique du Bénin (Akoègninou et al., 2006).

\section{Analyse des données}

Evaluation du niveau de connaissance de la pharmacopée vétérinaire

Le coefficient de corrélation de rang de Spearman a été calculé pour vérifier la présence ou l'absence de lien entre l'âge des enquêtés et le nombre de recettes rapportées et utilisées par chaque enquêté au seuil de $\alpha=5 \%$. Il se calcule par la formule suivante :

$$
r_{s}=1-\frac{6 \sum_{i=1}^{\mathrm{N}} \mathrm{d}_{\mathrm{i}}^{2}}{\mathrm{~N}^{3}-\mathrm{N}}
$$

où $\mathrm{d}$ est la différence entre les rangs des deux variables, $\mathrm{d}^{2}$ : le carré de $\mathrm{d}$ et $\mathrm{N}$ : le nombre d'individus d'échantillonnage.

Ici $\mathrm{N}=42$, la valeur $r_{\mathrm{s}}$ sera donc distribuée comme la valeur statistique $t$ de Student avec un degré de liberté de $\mathrm{N}-2$. La formule de $\mathrm{t}$ est la suivante :

$$
t=r_{s} \sqrt{\frac{\mathrm{N}-2}{1-r_{s}^{2}}}
$$

La signification de $r_{\mathrm{s}}$ sera celle de $t$. Lorsque la valeur absolue de $\mathrm{t}$ est inférieure à la valeur de $t$ tabulée au seuil de $\alpha=0,05$, la corrélation est non significative et on conclut une absence de lien entre l'âge et le nombre de recettes citées. Dans le cas contraire, la corrélation est significative et on tire comme conclusion une présence de lien entre l'âge et le nombre de recettes rapportées et utilisées par chaque enquêté. Dans ce dernier cas, une corrélation négative montre qu'au fur et à mesure que l'âge évolue, le nombre de recettes citées est petit alors qu'une corrélation positive montre qu'au fur et à mesure que l'âge évolue, le nombre de recettes citées est important.

Importance de la Flore à usages vétérinaires dans la zone cotonnière du Nord-Bénin Diversité des plantes à usages vétérinaires

La liste des espèces a été établie. La nomenclature botanique utilisée est celle de Akoègninou et al. (2006). La diversité floristique a été évaluée par le biais de la richesse spécifique, le nombre de familles et la richesse spécifique des familles.

Trois paramètres ont été déterminés. Il s'agit de la Fr, la Fcr et la Cpr.

La Fréquence des recettes $(\mathrm{Fr})$ et la Fréquence de citation de recettes (Fcr) ont permis d'identifier les recettes crédibles pour chaque maladie. Les recettes ayant les valeurs de Fr et de Frr les plus élevées sont considérées comme recettes crédibles. Des tests in vitro et in vivo sur les animaux seront réalisés par d'autres spécialistes.

Elles se calculent par les formules suivantes :

$$
\mathrm{Fr}=\frac{\mathrm{Ncr}}{\mathrm{N}} \times 100
$$

où Ncr est le nombre de citation d'une recette traitant une maladie donnée et $\mathrm{N}$ le nombre total de citation de toutes les recettes traitant la maladie. 


$$
\text { Fcr }=\frac{N c r}{N t} \times 100
$$

où Ncr est le nombre de citation d'une recette traitant une maladie donnée et Nt le nombre total de citation de toutes les recettes traitant toutes les maladies.

La Cpr est la contribution de chaque plante dans la constitution des recettes. C'est aussi la Fréquence d'implication des plantes dans les recettes. Elle a été calculée par la formule :

$$
\mathrm{C}_{\mathrm{pr}}=\frac{\mathrm{Nr}}{\mathrm{Nt}} \times 100
$$

où $\mathrm{Nr}$ le nombre de recettes sollicitant la plante et $\mathrm{Nt}$ le nombre total de recettes.

\section{Vulnérabilité des espèces}

Pour évaluer l'impact écologique de l'utilisation des plantes en pharmacopée vétérinaire, il a été déterminé la vulnérabilité de ces plantes. Les plantes herbeuses (érigées ou lianescentes) à reproduction par graine et les plantes annuelles (thérophytes) cultivées ne sont pas prises en compte dans cette étude car ne sont pas vulnérables du fait de leur mode de reproduction. Les espèces les plus vulnérables sont celles qui seront très populaires, qui croissent lentement, qui se reproduisent difficilement, qui habitent les milieux fragiles et qui, de plus, ont une distribution géographique très limitée (Okafor and Ham, 1999). Les paramètres d'évaluation de vulnérabilité à retenir sont ceux de Betti (2001): la popularité de l'espèce, l'organe végétal utilisé, le type morphologique, la fréquence de la plante dans le milieu et de Traoré al. (2011): le mode de collecte (Tableau 1). Pour chaque paramètre, l'échelle d'appréciation de la vulnérabilité est la suivante: 1 : espèce non vulnérable pour le paramètre considéré, 2 : espèce à vulnérabilité moyenne, 3 : espèce très vulnérable. Selon l'auteur, la prise en compte de façon combinée des différents paramètres permet de définir un indice de vulnérabilité $\left(\mathrm{I}_{\mathrm{v}}\right)$ propre à chaque espèce. Cet indice est obtenu en calculant la moyenne des différentes valeurs pour chaque espèce. Une espèce dont $I_{V}<2$ sera dite non vulnérable pour son usage en pharmacopée vétérinaire, une autre dont $2 \leq \mathrm{I}_{\mathrm{v}}$ $<2,5$ sera considérée comme vulnérable, aussi une espèce à $I_{v} \geq 2,5$ sera très vulnérable.

\section{Evaluation du consensus sur les phytoremèdes employés \\ Le Facteur de Consensus de} l'Informateur (FCI) a été calculé. Le Facteur de Consensus permet d'évaluer le degré d'homogénéité des informations données par les enquêtés (Andrade-Cetto and Heinrich, 2011). Il est calculé par la formule:

$$
\text { FCI }=\frac{\text { Ncu }- \text { Neu }}{\text { Ncu }-1}
$$

avec $\mathbf{N}_{\text {cu }}$ le nombre de citations d'usage rapporté dans chaque catégorie de maladies et $\mathrm{N}_{\mathrm{eu}}$ le nombre d'espèces utilisées pour le traitement des maladies. Il varie de 0 à 1 (Ugulu, 2009).

- La valeur 0 est le plus faible degré et correspond à des points de vue différents de la part des enquêtés dans l'utilisation des plantes pour traiter une catégorie de maladies ;

- les valeurs inférieures à 0,5 sont considérées comme faibles et désignent un consensus faible d'utilisation des plantes ;

- la valeur 0,5 est le degré moyen du facteur et indique un consensus moyen d'utilisation des plantes ;

- les valeurs comprises entre 0,5 et 1 sont les degrés relativement forts et montrent un accord relativement élevé dans l'utilisation des plantes pour traiter la catégorie de maladies ;

- et la valeur 1 est le plus fort degré montrant un consensus total dans l'utilisation des plantes pour traiter la catégorie de maladies.

Pour l'appliquer, les maladies ou affections et symptômes sont catégorisés selon le système ou l'appareil qui est atteint (Andrade-Cetto and Heinrich, 2011). Ainsi, les helminthoses, la diarrhée, etc., ont été classées dans le groupe des pathologies gastrointestinales (Tableau 2). 


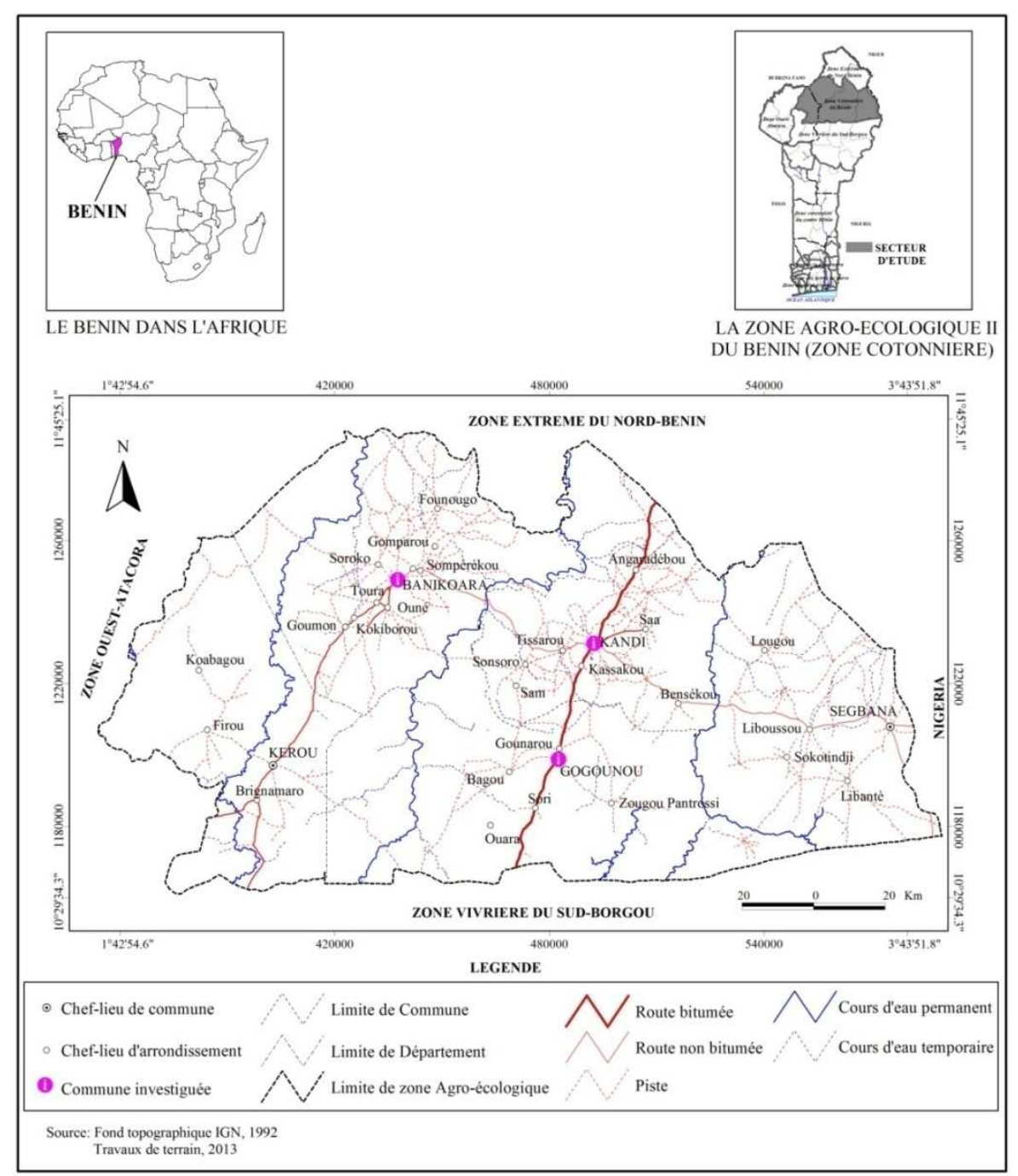

Figure 1 : Carte montrant la zone d'investigation au Nord-Bénin.

\section{RESULTATS}

\section{Caractéristiques socioculturelles des} enquêtés

L'âge moyen de la population enquêtée est de 48 ans. En tenant compte des classes d'âges, les individus ayant 40 - 49 ans sont majoritaires, avec une proportion de $38 \%$ de l'échantillon enquêté. Ils sont suivis des enquêtés ayant 50 - 59 ans $(27 \%), 60-69$ ans (15\%), $30-39$ ans (15\%), $70-79$ ans (3\%) et $0-29$ ans $(02 \%)$.

S'agissant de la répartition des enquêtés dans les groupes ethniques, les Peulh font $84 \%$ des enquêtés et constituent le groupe majoritaire. La succession dans l'ordre décroissant des autres groupes est le suivant : Bariba (12\%), Dendi et Gando (02\% chacun).

Importance de la flore à usages vétérinaires dans la zone cotonnière du Nord-Bénin et degré de connaissance des plantes à usages ethnovétérinaires

Diversité des plantes à usages vétérinaires

$\mathrm{Au}$ total, 56 espèces végétales à usages vétérinaires ont été recensées dans la zone cotonnière au Nord-Bénin. Elles sont reparties dans 50 genres et 22 familles. Les familles les plus importantes en termes de leur richesse spécifique sont: les Leguminosae (34\%), les Combretaceae (11\%) et les Meliaceae (07\%) 
(Tableau 3). En considérant le type morphologique, les arbres sont les plus fréquents avec un pourcentage de $61 \%$. Ils sont suivis des arbustes (21\%), des herbes dressées $(16 \%)$ et les lianes herbeuses $(02 \%)$. Sur les 56 espèces recensées, 4 seulement constituent des espèces cultivées, contre 52 espèces spontanées.

En tenant compte de la contribution des plantes dans la constitution des recettes, Khaya senegalensis est impliquée dans $21 \%$ des recettes. Ensuite, viennent Cassia sieberiana (07\%), Pterocarpus erinaceus, Vitellaria paradoxa (06\% chacune) et Balanites aegyptiaca (05\%). Au nombre des plantes ayant une contribution faible $(01 \%)$, on peut citer Lannea acida, Cussonia arborea, Afzelia africana, etc. (Tableau 3).

S'agissant du nombre de maladies traitées, Khaya senegalensis intervient dans le traitement de 7 maladies (Tableau 3). Parmi les plantes traitant une seule maladie, il y a Combretum glutinosum, Lagenaria siceraria, Swartzia madagascariensis, etc.

Les valeurs les plus importantes obtenues pour la Fr sont : $100 \%$ pour Carica papaya dans le traitement de la rétention placentaire, $80 \%$ pour Mitragyna inermis + Anogeissus leiocarpa dans le traitement de la Tuberculose et $61 \%$ pour Balanites aegyptiaca + Vigna unguiculata dans le traitement de l'agalactie (Tableau 4). Les valeurs faibles concernent Vitellaria paradoxa dans le traitement de la Pasteurellose avec 02\%; Acacia senegal cité comme antidiarrhéique avec 03\% (Tableau 4).

Le Tableau 4 indique aussi la fréquence de citation de recettes. Elle varie de $01 \%$ pour Pterocarpus erinaceus + Kigelia africana dans le traitement de la brucellose à $13 \%$ pour Balanites aegyptiaca + Vigna unguiculata dans le traitement de l'agalactie.

Le coefficient de corrélation de rang de Spearman calculé n'est pas significative et montre une absence de lien entre l'âge des enquêtés et le nombre de recettes rapportées et utilisées par chaque enquêté au seuil de $\alpha=$ $05 \%$ (corrélation de Spearman $=0,098 ; \mathrm{t}=$ $0,622 ; \mathrm{t}<2,021)$. Autrement dit, la connaissance ethnovétérinaire ne dépend pas de l'âge de l'enquêté.
La Figure 2 présente les fréquences de citation des organes végétaux. Elle indique que les écorces de tige sont les organes les plus cités avec un pourcentage de $61 \%$. Les autres organes (tige, chaume et graines) ne sont impliqués que dans $03 \%$ des recettes.

La préparation des produits végétaux entrant dans le traitement des pathologies fait intervenir 8 modes de préparation dont le plus fréquent est la décoction (49\%). Ensuite, viennent le pilage (23\%), la macération $(14 \%)$, la poudre $(10 \%)$ et les autres (trituration, cataplasme, calcination et torréfaction) font $04 \%$.

Cette étude s'est préoccupée aussi de l'origine de la connaissance ethnovétérinaire. Il s'est révélé que la source principale du savoir ethnovétérinaire est la Famille d'appartenance $(90 \%$ des éleveurs) contre seulement $10 \%$ pour les savoirs transmis d'éleveurs à éleveurs.

\section{Vulnérabilité des espèces}

$\mathrm{Au}$ total, 31 espèces végétales possèdent un indice de vulnérabilité supérieur ou égal à 2 et inférieur à 2,5 (Figure 3). Elles sont dites de ce fait espèces vulnérables. Cette flore vulnérable constitue $55 \%$ des espèces recensées. Les espèces comme Anogeissus leiocarpa, Cissus quadrangularis, Khaya senegalensis, Parkinsonia aculeata, Pterocarpus erinaceus et Ximenia americana sont les plus vulnérables avec un indice de vulnérabilité égal à 2,2.

En considérant les paramètres de vulnérabilité, les organes végétaux utilisés et leurs modes de collecte sont les paramètres qui ont une influence considérable sur la vulnérabilité des espèces. En effet, la coupe intervient dans $61 \%$ des cas de collecte. Ainsi beaucoup d'espèces sont écorcées (Khaya senegalensis, Prosopis africana, Ficus sycomorus...), abattues (Adenium obesum) et déracinées (Annona senegalensis, Parkinsonia aculeata, Sarcocephalus latifolius...). De l'autre côté, la cueillette d'organes intervient dans 39\% des cas de collecte. Les feuilles (Carica papaya, Guieria senegalensis, Cussonia arborea ...) et les fruits (Acacia nilotica ssp nilotica, Tamarindus indicus, Vitellaria paradoxa...) sont cueillis à la main. Enfin le ramassage intervient très peu dans les modes de collecte des organes végétaux avec 
une fréquence de $0,01 \%$. Dans ce cas, l'organe ramassé est le fruit (Azadirachta indica).

Notons que $30 \%$ de la flore recensée sont non vulnérables (indice inférieur à 2) parmi lesquelles, on distingue Balanites aegyptiaca, Mitragyna inermis, Combretum glutinosum, etc. (Figure 3).

\section{Affections animales dans la zone cotonnière du Nord-Bénin Maladies recensées}

En considérant la fréquence de citation, la Fièvre aphteuse et la Pasteurellose sont les maladies les plus fréquentes avec des pourcentages respectifs de $19 \%$ et $17 \%$ (Figure 4).
Les affections telles que l'avortement, la coccidiose, et la météorisation sont très faiblement citées.

Evaluation du consensus sur les phytoremèdes employés

Les maladies sont classées en 5 catégories selon l'appareil atteint. Les valeurs $\mathrm{du}$ Facteur de consensus de l'Informateur calculé pour chacune d'elles varient de 0,4 pour les affections sanguines à 0,5 pour les affections respiratoires (Tableau 5). Le nombre d'espèces utilisées dans le traitement de ces catégories de maladies est compris entre 14 pour les affections obstétricales et 31 pour les affections respiratoires.

Tableau 1 : Importance des paramètres impliqués dans l'évaluation de la vulnérabilité.

\begin{tabular}{|c|c|c|c|}
\hline \multirow{2}{*}{ Paramètres } & \multicolumn{3}{|c|}{ Vulnérabilité à exploitation incontrôlée } \\
\hline & Faible (échelle $=1$ ) & Moyenne (échelle = 2) & Forte (échelle $=3$ ) \\
\hline Popularité & faible: $\mathrm{Cpr}<20 \%$ & moyenne $20 \leq \mathrm{Cpr}<60$ & forte $\mathrm{Cpr} \geq 60$ \\
\hline Organes végétaux & feuilles et sève & fruit & $\begin{array}{c}\text { écorce de tige, racines, } \\
\text { graines, tige }\end{array}$ \\
\hline Type morphologique & arbre, arbuste & liane ligneuse & herbe, liane herbeuse \\
\hline $\begin{array}{lll}\begin{array}{l}\text { Fréquence } \\
\text { milieu }\end{array} & & \text { dans } \\
\end{array}$ & Abondant & moins abondant & Rare \\
\hline Mode de collecte & Ramassage & & cueillette, coupe \\
\hline
\end{tabular}

Tableau 2 : Catégorisation des maladies et symptômes animaux selon l'appareil de l'animal atteint.

\begin{tabular}{ll}
\hline Catégories de maladies & Maladies \\
\hline Affections sanguines & $\begin{array}{l}\text { Newcastle, Piroplasmose, Trypanosomose, Charbon } \\
\text { symptomatique }\end{array}$ \\
\hline Affections cutanées & $\begin{array}{l}\text { Gale, Variole, Dermatophilose, Plaies, Fièvre aphteuse, } \\
\text { Ectoparasitoses, Morsure de serpent }\end{array}$ \\
\hline $\begin{array}{l}\text { Pathologies gastro- } \\
\text { intestinales }\end{array}$ & Diarrhée, Helminthoses, Fièvre aphteuse, Météorisation \\
\hline Affections respiratoires & PPCB, Pasteurellose, Toux, Tuberculose, Variole \\
\hline $\begin{array}{l}\text { Affections obstétricales ou } \\
\text { de la reproduction et autre }\end{array}$ & Brucellose, Rétention placentaire, Agalactie, Tétanie d'herbage \\
\hline
\end{tabular}


Tableau 3 : Noms scientifiques et vernaculaires des plantes à usages vétérinaires recensées, les familles botaniques et leurs fréquences, et la contribution des plantes.

\begin{tabular}{|c|c|c|c|c|c|c|c|}
\hline Noms scientifiques & $\begin{array}{c}\text { Noms } \\
\text { vernaculaires }\end{array}$ & $\begin{array}{c}\text { Familles } \\
\text { botaniques }\end{array}$ & FF & Org & TM & $\mathbf{N m}$ & $\begin{array}{c}\text { Cpr en } \\
\%\end{array}$ \\
\hline Lannea acida A.Rich. & Tchami $^{1}$ & \multirow{2}{*}{ Anacardiaceae } & \multirow{2}{*}{3} & $\mathrm{E}$ & $\overline{\mathrm{A}}$ & 2 & 1 \\
\hline Mangifera indica $\mathrm{L}$. & Mangohi $^{1}$ & & & $\mathrm{E}$ & $\mathrm{A}$ & 1 & 5 \\
\hline $\begin{array}{l}\text { Annona senegalensis } \\
\text { Pers. }\end{array}$ & Doukolhi $^{1}$ & Annonaceae & 2 & $\mathrm{R}$ & $\mathrm{Ar}$ & 2 & 1 \\
\hline $\begin{array}{l}\text { Adenium obesum } \\
\text { (Forsk.) Roem. \& } \\
\text { Schult. }\end{array}$ & Djolouhi $^{1}$ & Apocynaceae & 2 & $\mathrm{~F}, \mathrm{~T}$ & $\mathrm{Ar}$ & 2 & 2 \\
\hline $\begin{array}{l}\text { Cussonia arborea } \\
\text { Hochst.ex A. Rich. }\end{array}$ & $\begin{array}{c}\text { Alanbélouwa }^{1} \text { ou } \\
\text { tchémoura }^{1}\end{array}$ & Araliaceae & 2 & $\mathrm{~F}$ & A & 1 & 1 \\
\hline $\begin{array}{l}\text { Kigelia africana } \\
\text { (Lam.) Benth. }\end{array}$ & Djilidjalahi $^{1}$ & Bignoniaceae & 2 & Fr, E & A & 3 & 5 \\
\hline Adansonia digitata $\mathrm{L}$. & Bokki $^{1}$ & \multirow[b]{2}{*}{ Bombacaceae } & \multirow[b]{2}{*}{3} & Fr, E & $\mathrm{A}$ & 2 & 4 \\
\hline $\begin{array}{l}\text { Bombax costatum } \\
\text { Pellegr.\& Vuillet. }\end{array}$ & Kourouhi $^{1}$ & & & Ex, E & A & 2 & 5 \\
\hline Carica papaya $\mathrm{L}$. & Karabossi $^{1}$ & Caricaceae & 2 & $\mathrm{~F}$ & $\mathrm{H}$ & 1 & 1 \\
\hline $\begin{array}{l}\text { Anogeissus leiocarpa } \\
\text { (DC.) Guil. \& Perr. }\end{array}$ & Kodjoli $^{1}$, Ganahi $^{1}$ & \multirow{6}{*}{ Combretaceae } & \multirow{6}{*}{11} & $\mathrm{~F}, \mathrm{E}$ & A & 4 & 5 \\
\hline $\begin{array}{l}\text { Combretum collinum } \\
\text { Fresen. }\end{array}$ & Lactibodehi $^{1}$ & & & F, E, R & A & 2 & 2 \\
\hline $\begin{array}{l}\text { Combretum } \\
\text { glutinosum Perr.ex } \\
\text { DC. }\end{array}$ & Dooki ${ }^{1}$ & & & $\mathrm{E}$ & $\mathrm{A}$ & 1 & 1 \\
\hline $\begin{array}{l}\text { Combretum molle } \\
\text { R.Br.ex G.Don. }\end{array}$ & Gnangnankoudohi ${ }^{1}$ & & & $\mathrm{E}$ & A & 1 & 2 \\
\hline $\begin{array}{l}\text { Guiera senegalensis } \\
\text { J.F.Gmel. }\end{array}$ & Yoloko $^{2}$ & & & $\mathrm{~F}$ & $\mathrm{Ar}$ & 2 & 1 \\
\hline $\begin{array}{l}\text { Pteleopsis suberosa } \\
\text { Engl.\& Diels. }\end{array}$ & $\begin{array}{c}\text { Bounou-bounou }^{2} \text { ou } \\
\text { Padji-bohili }^{1}\end{array}$ & & & $\mathrm{~F}, \mathrm{E}$ & $\mathrm{Ar}$ & 2 & 2 \\
\hline $\begin{array}{l}\text { Lagenaria siceraria } \\
\text { (Molina) Standl. }\end{array}$ & Wingnarou $^{2}$ & Cucurbitaceae & 2 & $\mathrm{Fr}$ & $\mathrm{H}$ & 1 & 1 \\
\hline $\begin{array}{l}\text { Aganope stuhlmannii } \\
\text { (Taub.) Adema. }\end{array}$ & Yandorou $^{2}$ & \multirow[t]{3}{*}{ Leguminosae } & \multirow[t]{3}{*}{34} & $\mathrm{E}$ & A & 2 & 2 \\
\hline $\begin{array}{l}\text { Acacia polyacantha } \\
\text { Willd. }\end{array}$ & Patouki $^{1}$ & & & $\mathrm{R}$ & $\mathrm{A}$ & 3 & 4 \\
\hline $\begin{array}{l}\text { Acacia senegal (L.) } \\
\text { Willd. }\end{array}$ & - & & & Fr, E & A & 1 & 2 \\
\hline
\end{tabular}




\begin{tabular}{|c|c|c|c|c|c|c|c|}
\hline $\begin{array}{l}\text { Acacia nilotica (L.) } \\
\text { Willd. ex Delile ssp. } \\
\text { nilotica }\end{array}$ & Gadé $^{1}$ & & & Fr, F & A & 1 & 1 \\
\hline Afzelia Africana Sum. & Wargnahi $^{1}$ & & & $\mathrm{~F}, \mathrm{E}$ & $\mathrm{A}$ & 2 & 1 \\
\hline $\begin{array}{l}\text { Albizia chevalieri } \\
\text { Harrms }\end{array}$ & Sabi-samporo $^{1}$ & & & $\mathrm{R}$ & A & 1 & 2 \\
\hline $\begin{array}{l}\text { Cassia sieberiana } \\
\text { DC. }\end{array}$ & $\begin{array}{c}\text { Guiédanéhi }{ }^{1} \text { ou } \\
\text { kohi }^{1}\end{array}$ & & & $\mathrm{E}$ & A & 3 & 7 \\
\hline $\begin{array}{l}\text { Daniellia oliveri } \\
\text { (Rolfe) Hutch.\& } \\
\text { Dalziel }\end{array}$ & Karlahi $^{1}$ & & & $\mathrm{E}$ & A & 1 & 4 \\
\hline $\begin{array}{l}\text { Detarium } \\
\text { microcarpum Gill. \& } \\
\text { Perr. }\end{array}$ & Konkéhi $^{1}$ & & & $\mathrm{~F}, \mathrm{E}$ & A & 3 & 2 \\
\hline $\begin{array}{l}\text { Isoberlinia doka } \\
\text { Craib \& Stapf }\end{array}$ & Batahi $^{1}$ & & & $\mathrm{~T}$ & A & 1 & 1 \\
\hline $\begin{array}{l}\text { Parkia biglobosa } \\
\text { (Jacq.) R.Br. ex } \\
\text { Benth. }\end{array}$ & Naréhi & & & Fr, E, R & A & 4 & 5 \\
\hline $\begin{array}{l}\text { Parkinsonia aculeata } \\
\text { L. }\end{array}$ & Bonssangounian $^{2}$ & & & $\mathrm{R}$ & $\mathrm{Ar}$ & 1 & 1 \\
\hline $\begin{array}{l}\text { Pericopsis laxiflora } \\
\text { (Benth.ex Baker) }\end{array}$ & $\begin{array}{c}\text { Cinanfégou }^{2} \text { ou } \\
\text { sorokouhi }^{1}\end{array}$ & & & $\mathrm{R}, \mathrm{E}$ & A & 2 & 3 \\
\hline $\begin{array}{l}\text { Piliostigma thonningii } \\
\text { (Schumach.) Milne- } \\
\text { Redh. }\end{array}$ & Barkéhi $^{1}$ & & & $\mathrm{E}$ & $\mathrm{Ar}$ & 1 & 1 \\
\hline $\begin{array}{l}\text { Prosopis africana } \\
\text { (Gill.\& Perr.) Taub. }\end{array}$ & Kohi $^{1}$ & & & $\mathrm{E}$ & A & 2 & 3 \\
\hline $\begin{array}{l}\text { Pterocarpus } \\
\text { erinaceus Poir. }\end{array}$ & Banouhi $^{1}$ & & & $\mathrm{~F}, \mathrm{E}$ & A & 4 & 6 \\
\hline $\begin{array}{l}\text { Swartzia } \\
\text { madagascariensis } \\
\text { Desv. }\end{array}$ & Kokobi $^{1}$ & & & $\mathrm{R}$ & $\mathrm{Ar}$ & 1 & 1 \\
\hline Tamarindus indica L. & Djatami $^{1}$ & & & Fr & $\mathrm{Ar}$ & 1 & 1 \\
\hline $\begin{array}{l}\text { Vigna unguiculata } \\
\text { (L.) Walp. }\end{array}$ & Yeinbê $^{1}$ & & & $\mathrm{Fr}$ & $\mathrm{H}$ & 1 & 3 \\
\hline $\begin{array}{l}\text { Strychnos spinosa } \\
\text { Lam. }\end{array}$ & Mapantarahi $^{1}$ & Loganiaceae & 2 & $\mathrm{R}$ & $\mathrm{Ar}$ & 1 & 2 \\
\hline $\begin{array}{l}\text { Azadirachta indica } \\
\text { A.Juss. }\end{array}$ & Millii $^{1}$ & Meliaceae & 7 & G & A & 1 & 1 \\
\hline Khaya senegalensis & $\mathrm{Kahi}^{1}$ & & & $\mathrm{~F}, \mathrm{E}$ & $A$ & 7 & 1 \\
\hline
\end{tabular}




\begin{tabular}{|c|c|c|c|c|c|c|c|}
\hline \multicolumn{2}{|l|}{ (Desr.) A. Juss. } & & & \multirow{4}{*}{$\mathrm{E}$} & \multirow{4}{*}{ A } & \multirow{4}{*}{3} & \multirow{4}{*}{2} \\
\hline Pseudocedrela & & & & & & & \\
\hline kotschyi (Schweinf.) & Kahi-loumi $^{1}$ & & & & & & \\
\hline \multicolumn{4}{|l|}{ Harms. } & & & & \\
\hline $\begin{array}{l}\text { Trichillia emetica } \\
\text { Vahl }\end{array}$ & Pissa-ladè $^{1}$ & & & $\mathrm{E}$ & $\mathrm{A}$ & 1 & 1 \\
\hline Ficus sycomorus L. & $\mathrm{Ibi}^{1}$ & \multirow[b]{2}{*}{ Moraceae } & \multirow[b]{2}{*}{3} & $\mathrm{E}$ & $\mathrm{A}$ & 2 & 1 \\
\hline $\begin{array}{l}\text { Ficus platyphylla } \\
\text { Delile }\end{array}$ & Doundéhi ${ }^{1}$ & & & $\mathrm{E}$ & $\mathrm{A}$ & 1 & 2 \\
\hline Ximenia americana $\mathrm{L}$. & $\begin{array}{l}\text { Tchapoulahi }{ }^{1} \text { ou } \\
\text { sètouèhi }^{1}\end{array}$ & Olacaceae & 2 & $\mathrm{R}$ & $\mathrm{Ar}$ & 1 & 2 \\
\hline $\begin{array}{l}\text { Ceratotheca } \\
\text { sesamoides Endl }\end{array}$ & Wari $^{2}$ & Pedaliaceae & 2 & $\mathrm{~F}$ & $\mathrm{H}$ & 1 & 1 \\
\hline $\begin{array}{l}\text { Oxytenanthera } \\
\text { abyssinica } \\
\text { (A.Rich.)Munro }\end{array}$ & - & \multirow{3}{*}{ Poaceae } & \multirow{3}{*}{5} & $\mathrm{~F}$ & $\mathrm{H}$ & 1 & 1 \\
\hline $\begin{array}{l}\text { Sorghum bicolor }(\mathrm{L} .) \\
\text { Moench }\end{array}$ & - & & & $\mathrm{Fr}$ & $\mathrm{H}$ & 1 & 1 \\
\hline Zea mays L. & Kokoniri $^{1}$ & & & Fr & $\mathrm{H}$ & 1 & 2 \\
\hline $\begin{array}{l}\text { Crossopteryx } \\
\text { febrifuga (G.Don) } \\
\text { Benth. }\end{array}$ & Rimadjogahi $^{1}$ & \multirow{3}{*}{ Rubiaceae } & \multirow{3}{*}{5} & Fr, F, E & $\mathrm{Ar}$ & 2 & 2 \\
\hline $\begin{array}{l}\text { Mitragyna inermis } \\
\text { (Willd.) Kuntze }\end{array}$ & Kooli $^{1}$ & & & $\mathrm{~F}, \mathrm{E}$ & A & 2 & 2 \\
\hline $\begin{array}{l}\text { Sarcocephalus } \\
\text { latifolius (Sm.) } \\
\text { E.A.Bruce }\end{array}$ & Barkouréhi ${ }^{1}$ & & & $\mathrm{R}$ & $\mathrm{Ar}$ & 2 & 2 \\
\hline $\begin{array}{l}\text { Vitellaria paradoxa } \\
\text { C.F.Gaertn. }\end{array}$ & Karéhi $^{1}$ & Sapotaceae & 2 & $\mathrm{Fr}, \mathrm{E}, \mathrm{F}$ & A & 4 & 6 \\
\hline Capsicum annuиm L. & Takin $^{2}$ & \multirow{2}{*}{ Solanaceae } & \multirow{2}{*}{3} & Fr & $\mathrm{H}$ & 1 & 1 \\
\hline Nicotiana tabacum L. & $\mathrm{Taba}^{2}$ & & & $\mathrm{~F}$ & $\mathrm{H}$ & 1 & 1 \\
\hline Vitex doniana Sweet & Gounmé $^{1}$ & Verbenaceae & 2 & $\mathrm{E}$ & $\mathrm{A}$ & 1 & 1 \\
\hline $\begin{array}{l}\text { Cissus } \\
\text { quadrangularis L. }\end{array}$ & Takounin-téri $^{3}$ & Vitaceae & 2 & $\mathrm{~T}$ & $\mathrm{~L}$ & 1 & 1 \\
\hline $\begin{array}{l}\text { Balanites aegyptiaca } \\
\text { (L.) Delile }\end{array}$ & $\operatorname{Tani}^{1}$ ou Tané ${ }^{1}$ & Zygophyllaceae & 2 & $\mathrm{E}$ & A & 2 & 5 \\
\hline
\end{tabular}


Tableau 4 : Maladies animales, mode de traitement, fréquence des recettes et fréquence de citation des recettes.

\begin{tabular}{|c|c|c|c|c|c|}
\hline \multirow{2}{*}{ Maladies } & \multicolumn{3}{|c|}{ Recettes } & \multicolumn{2}{|c|}{ Paramètres } \\
\hline & Composition & $\begin{array}{c}\text { Mode de } \\
\text { préparation }\end{array}$ & $\begin{array}{c}\text { Dose, posologie durée de } \\
\text { traitement et voie } \\
\text { d'administration }\end{array}$ & $\mathbf{F r}$ & Fer \\
\hline \multirow[t]{5}{*}{ Agalactie } & $\begin{array}{l}\text { écorce de Balanites aegyptiaca }+ \\
\text { fruits de Vigna unguiculata }+ \text { eau }\end{array}$ & Pilage + eau & $1 \mathrm{~L} /$ jour pendant 5 jours, per os & 61 & 13 \\
\hline & $\begin{array}{l}\text { racine de Swartzia } \\
\text { madagascariensis }+ \text { fruits de } \\
\text { Vigna unguiculata }+ \text { eau }\end{array}$ & pilage + eau & $1 \mathrm{~L} /$ jour pendant 5 jours, per os & 22 & 3 \\
\hline & $\begin{array}{l}\text { fruits de Lagenaria siceraria }+ \\
\text { coque du fruit de Sorghum bicolor } \\
+ \text { sel }\end{array}$ & décoction & $\begin{array}{l}\text { à volonté, } 2 \text { fois/ jour } \\
\text { pendant } 2 \text { jours, per os }\end{array}$ & 6 & 1 \\
\hline & $\begin{array}{l}\text { feuilles d'Adenium obesum + fruits } \\
\text { de Vigna unguiculata }\end{array}$ & pilage + eau & $\begin{array}{l}1 \mathrm{~L}, 2 \text { fois / jour pendant } 5 \text { jours, } \\
\text { per os }\end{array}$ & 6 & 1 \\
\hline & écorce de Balanites aegyptiaca & décoction & $\begin{array}{l}1 \mathrm{~L} \text { adultes et } 1 / 2 \mathrm{~L} \text { jeunes, } 2 \text { fois/ } \\
\text { jour jusqu'à guérison, per os }\end{array}$ & 5 & 1 \\
\hline \multirow[t]{2}{*}{ Brucellose } & écorce de Kigelia africana & décoction & $\begin{array}{l}1 \mathrm{~L} \text { adultes et } 1 / 2 \mathrm{~L} \text { jeunes, } 2 \text { fois/ } \\
\text { jour pendant une semaine, per os }\end{array}$ & 50 & 1 \\
\hline & $\begin{array}{l}\text { écorce de Pterocarpus erinaceus et } \\
\text { de Kigelia africana }\end{array}$ & décoction & $\begin{array}{l}1 \mathrm{~L} \text { adultes et } 1 / 2 \mathrm{~L} \text { jeunes, } 2 \text { fois / } \\
\text { jour pendant une semaine, per os }\end{array}$ & 50 & 1 \\
\hline $\begin{array}{l}\text { Charbon } \\
\text { symptomatique }\end{array}$ & écorce d'Aganope stulhmannii & décoction & $\begin{array}{l}1 \mathrm{~L} \text { adultes et } 1 / 2 \mathrm{~L} \text { jeunes, } 3 \text { fois/ } \\
\text { jour jusqu'à guérison, per os }\end{array}$ & 100 & 2 \\
\hline \multirow[t]{8}{*}{ Diarrhée } & écorce de Combretum molle & décoction & $\begin{array}{l}1 \mathrm{~L} \text { adultes et } 1 / 2 \mathrm{~L} \text { jeunes, } 2 \text { fois } \\
\text { en } 1 \text { jour, per os }\end{array}$ & 13 & 3 \\
\hline & écorce de Daniellia oliveri & décoction & $\begin{array}{l}1 \mathrm{~L} \text { adultes et } 1 / 2 \mathrm{~L} \text { jeunes, } 2 \text { fois/ } \\
\text { jour, pendant } 2 \text { jours, per os }\end{array}$ & 9 & 2 \\
\hline & écorce de Ficus platyphylla & décoction & $\begin{array}{l}1 \mathrm{~L} \text { adultes et } 1 / 2 \mathrm{~L} \text { jeunes, } 2 \text { fois / } \\
\text { jour, pendant } 2 \text { jours, per os }\end{array}$ & 3 & 1 \\
\hline & $\begin{array}{l}\text { écorces de Balanites aegyptiaca et } \\
\text { de Mitragyna inermis }\end{array}$ & décoction & $1 / 2 \mathrm{~L}, 1$ fois en 1 jour, per os & 4 & 1 \\
\hline & feuilles d'Anogeissus leiocarpa & pilage + eau & $\begin{array}{l}1 \mathrm{~L} \text { adultes et } 1 / 2 \mathrm{~L} \text { jeunes, } 2 \text { fois / } \\
\text { jour, jusqu'à guérison, per os }\end{array}$ & 13 & 3 \\
\hline & écorce de Vitex doniana & $\begin{array}{l}\text { Pilage }+ \text { eau } \\
\text { et tamisage }\end{array}$ & $\begin{array}{l}\text { à volonté, } 1 \text { fois / jour, } 2 \text { jours, per } \\
\text { os }\end{array}$ & 4 & 1 \\
\hline & fruits ou feuilles d'Acacia senegal & pilage & $\begin{array}{l}1 / 4 \mathrm{~L} \text { jeunes et } 1 / 2 \mathrm{~L} \text { adultes en } 1 \\
\text { jour }\end{array}$ & 3 & 1 \\
\hline & $\begin{array}{l}\text { écorce ou feuilles de Pteleopsis } \\
\text { suberosa et racines de Ximenia } \\
\text { americana }\end{array}$ & $\begin{array}{l}\text { poudre }+ \\
\text { eau }\end{array}$ & $\begin{array}{l}1 \mathrm{~L} \text { adultes et } 1 / 2 \mathrm{~L} \text { jeunes, } 2 \text { fois / } \\
\text { jour, jusqu'à guérison, per os }\end{array}$ & 4 & 1 \\
\hline
\end{tabular}


H. G. DASSOU et al. / Int. J. Biol. Chem. Sci. 8(1): 189-210, 2014

\begin{tabular}{|c|c|c|c|c|c|}
\hline & feuilles de Khaya senegalensis & décoction & $\begin{array}{l}1 \mathrm{~L} \text { adultes et } 1 / 2 \mathrm{~L} \text { jeunes, } 2 \text { fois / } \\
\text { jour, pendant } 5 \text { jours, per os }\end{array}$ & 9 & 2 \\
\hline & feuilles Cussonia arborea & décoction & $\begin{array}{l}1 \mathrm{~L} \text { adultes et } 1 / 2 \mathrm{~L} \text { jeunes, } 3 \text { fois / } \\
\text { jour, pendant } 3 \text { jours, per os }\end{array}$ & 9 & 3 \\
\hline & $\begin{array}{l}\text { feuilles d'Acacia polyacantha et de } \\
\text { Cussonia arborea }\end{array}$ & pilage + eau & $\begin{array}{l}1 \mathrm{~L} \text { adultes et } 1 / 2 \mathrm{~L} \text { jeunes, } 1 \text { fois / } \\
\text { jour, jusqu'à guérison, per os }\end{array}$ & 4 & 1 \\
\hline & $\begin{array}{l}\text { racine de Ximenia americana }+ \\
\text { écorce de Lannea acida }\end{array}$ & décoction & $\begin{array}{l}1 \mathrm{~L} \text { adultes et } 1 / 2 \mathrm{~L} \text { jeunes, } 1 \text { fois / } \\
\text { jour, pendant } 3 \text { jours, per os }\end{array}$ & 9 & 3 \\
\hline & racine de Combretum collinum & macération & $\begin{array}{l}1 \mathrm{~L} \text { adultes et } 1 / 2 \mathrm{~L} \text { jeunes, } 2 \text { fois / } \\
\text { jour, pendant } 2 \text { jours, per os }\end{array}$ & 4 & 1 \\
\hline & feuilles d'Adansonia digitata & trituration & 2 cuillérées, 2 fois en jour, per os & 4 & 1 \\
\hline & écorce de Ficus sycomorus & décoction & $\begin{array}{l}3 \text { verres bambou, } 2 \text { fois / jour, } \\
\text { pendant une semaine, per os }\end{array}$ & 4 & 1 \\
\hline Dermatophilose & fruits de Zea mays + ampicilline & macération & $\begin{array}{l}\text { quelques centilitres une fois par } \\
\text { injection }\end{array}$ & 100 & 1 \\
\hline \multirow[t]{2}{*}{ Ectoparasitoses } & $\begin{array}{l}\text { fruits de Vitellaria paradoxa }+ \\
\text { essence et savon }\end{array}$ & $\begin{array}{l}\text { chauffage et } \\
\text { pilage }\end{array}$ & $\begin{array}{l}\text { à la taille de la partie atteinte, } \\
2 \text { fois /jour, jusqu'à guérison, } \\
\text { per cutanée }\end{array}$ & 25 & 1 \\
\hline & graine d'Azadirachta indica & pilage & $\begin{array}{l}\text { à la taille de la partie atteinte, } \\
2 \text { fois /jour, jusqu'à guérison, } \\
\text { per cutanée }\end{array}$ & 75 & 4 \\
\hline \multirow[t]{8}{*}{ Fièvre aphteuse } & $\begin{array}{l}\text { fruit ou écorce d'Acacia nilotica } \\
\text { ssp. nilotica }\end{array}$ & $\begin{array}{l}\text { décoction ou } \\
\text { macération }\end{array}$ & $\begin{array}{l}\text { petite quantité, } 1 \text { fois / jour pendant } \\
\text { une semaine, per os }\end{array}$ & 27 & 6 \\
\hline & $\begin{array}{l}\text { écorce de Vitellaria paradoxa et de } \\
\text { Pterocarpus erinaceus + racine } \\
\text { d'Acacia polyacantha }\end{array}$ & décoction & $\begin{array}{l}1 \mathrm{~L} \text { adultes et } 1 / 2 \mathrm{~L} \text { jeunes, } 1 \text { fois / } \\
\text { jour pendant } 3 \text { jours, per os }\end{array}$ & 5 & 1 \\
\hline & écorce d'Anogeissus leiocarpa & poudre & $\begin{array}{l}\text { à volonté, } 1 \text { fois en } 2 \text { jours pendant } \\
\text { une semaine, per os }\end{array}$ & 5 & 1 \\
\hline & $\begin{array}{l}\text { écorces de Khaya senegalensis, de } \\
\text { Lannea acida et de Pterocarpus } \\
\text { erinaceus + urine de bœuf }\end{array}$ & macération & $\begin{array}{l}\text { petite quantité, } 2 \text { fois / jour, jusqu'à } \\
\text { guérison, per os et per cutanée }\end{array}$ & 16 & 3 \\
\hline & $\begin{array}{l}\text { feuilles de Detarium microcarpum } \\
+ \text { sel }\end{array}$ & pilage & $\begin{array}{l}\text { petite quantité, } 1 \text { fois / jour, } \\
\text { pendant } 3 \text { jours, per os }\end{array}$ & 5 & 1 \\
\hline & $\begin{array}{l}\text { écorce de Balanites aegypticaca }+ \\
\text { sel }\end{array}$ & poudre & $\begin{array}{l}\text { petite quantité, } 2 \text { fois / jour, jusqu'à } \\
\text { guérison, per os }\end{array}$ & 32 & 7 \\
\hline & fruits de Kigelia africana & décoction & $\begin{array}{l}1 / 2 \mathrm{~L} \text { par sujet/ jour, pendant } 3 \\
\text { jours, per os }\end{array}$ & 5 & 1 \\
\hline & écorce de Cassia sieberiana & décoction & $\begin{array}{l}\text { petite quantité, } 1 \text { fois /jour, pendant } \\
3 \text { jours, per os }\end{array}$ & 5 & 1 \\
\hline Gale & feuilles de Ceratotheca sesamoides & $\begin{array}{l}\text { pilage et } \\
\text { décoction }\end{array}$ & $\begin{array}{l}2 \mathrm{~L} \text { adultes et } 1 \mathrm{~L} \text { jeunes, } 1 \text { fois / } \\
\text { jour, pendant } 2 \text { jours, per os }\end{array}$ & 100 & 1 \\
\hline
\end{tabular}


H. G. DASSOU et al. /Int. J. Biol. Chem. Sci. 8(1): 189-210, 2014

\begin{tabular}{|c|c|c|c|c|c|}
\hline \multirow[t]{6}{*}{ Helminthoses } & écorce de Khaya senegalensis & poudre & $\begin{array}{l}\text { quelques cuillérées, } 2 \text { fois/jour } \\
\text { pendant } 3 \text { jours, per os }\end{array}$ & 36 & 1 \\
\hline & écorce de Cassia sieberiana, de & \multirow{4}{*}{ poudre } & \multirow{4}{*}{$\begin{array}{l}\text { petite quantité, } 1 \text { fois / jour pendant } \\
\text { une semaine, per os }\end{array}$} & \multirow{4}{*}{30} & \multirow{4}{*}{1} \\
\hline & Prosopis africana et de Khaya & & & & \\
\hline & senegalensis + chaume de & & & & \\
\hline & Sorghum bicolor + sel & & & & \\
\hline & feuilles d'Anogeissus leiocarpa & $\begin{array}{l}\text { pilage et } \\
\text { trituration }\end{array}$ & $\begin{array}{l}1 \mathrm{~L} \text { adultes et } 1 / 2 \mathrm{~L} \text { jeunes, } 2 \text { fois / } \\
\text { jour pendant } 3 \text { jours, per os }\end{array}$ & 34 & 1 \\
\hline Météorisation & fruits de Parkia biglobosa & $\begin{array}{l}\text { poudre }+ \\
\text { eau }\end{array}$ & $\begin{array}{l}1 / 2 \mathrm{~L}, 1 \text { fois /jour, jusqu'à guérison, } \\
\text { per os }\end{array}$ & 100 & 1 \\
\hline $\begin{array}{l}\text { Morsure de } \\
\text { serpent }\end{array}$ & racines de Combretum glutinosum & décoction & $\begin{array}{l}1 \mathrm{~L} \text { adultes et } 1 / 2 \mathrm{~L} \text { jeunes, } 1 \text { fois/ } \\
\text { jour pendant } 3 \text { jours, per os }\end{array}$ & 100 & 2 \\
\hline \multirow[t]{9}{*}{ Newcastle } & racine d'Annona senegalensis + & macération & \multirow{2}{*}{ à volonté, jusqu'à guérison, per os } & \multirow[t]{2}{*}{8} & \multirow[t]{2}{*}{1} \\
\hline & fruits de Capsicum annuиm & + pilage & & & \\
\hline & $\begin{array}{l}\text { écorces de Khaya senegalensis et } \\
\text { de Cassia sieberiana }\end{array}$ & macération & $\begin{array}{l}\text { 1/2 à } 1 \mathrm{~L} \text {, à volonté, jusqu'à } \\
\text { guérison, per os }\end{array}$ & 7 & 1 \\
\hline & tige de Cissus quadrangularis & macération & à volonté, jusqu'à guérison, per os & 9 & 1 \\
\hline & écorce de Pericopsis laxiflora & décoction & à volonté, jusqu'à guérison, per os & 8 & 1 \\
\hline & feuilles de Nicotiana tabacum & macération & à volonté, jusqu'à guérison, per os & 7 & 1 \\
\hline & écorce de Khaya senegalensis & macération & à volonté, jusqu'à guérison, per os & 7 & 1 \\
\hline & $\begin{array}{l}\text { écorces de Khaya senegalensis et } \\
\text { de Pseudocedrela kotschyi }\end{array}$ & macération & à volonté, jusqu'à guérison, per os & 31 & 5 \\
\hline & $\begin{array}{l}\text { écorces de Parkia biglobosa et de } \\
\text { Pseudocedrela kotschyi }\end{array}$ & macération & à volonté, jusqu'à guérison, per os & 23 & 3 \\
\hline \multirow{9}{*}{$\begin{array}{l}\text { Pasteurellose } \\
\text { bovine }\end{array}$} & écorces de Khaya senegalensis, de & & & & \\
\hline & Cassia sieberiana et de Mangifera & décoction & fois/jour, jusqu'à guérison, per os & 23 & 11 \\
\hline & $\begin{array}{l}\text { écorces de Khaya senegalensis et } \\
\text { d'Adansonia digitata }+ \text { sel }\end{array}$ & Poudre & $\begin{array}{l}\text { à volonté, } 1 \text { fois / jour pendant une } \\
\text { semaine, per os }\end{array}$ & 3 & 1 \\
\hline & $\begin{array}{l}\text { écorce de Crossopteryx febrifuga }+ \\
\text { racine d'Albizia chevalieri }\end{array}$ & décoction & $1 \mathrm{~L} /$ jour jusqu'à guérison, per os & 2 & 1 \\
\hline & racines d'Albiza chevalieri et de & & & & \\
\hline & $\begin{array}{l}\text { Sarcocephalus latifolius + écorces } \\
\text { de Pseudocedrela kotschyi, de }\end{array}$ & décoction & $1 \mathrm{~L}$ adultes et $1 / 2 \mathrm{~L}$ jeunes, 1 fois & 5 & 2 \\
\hline & Khaya senegalensis, de Bombax & & & & \\
\hline & costatum et de Ficus sycomorus & & & & \\
\hline & $\begin{array}{l}\text { exsudat de Bombax costatum + } \\
\text { tige Adenium obesum + écorce } \\
\text { d'Isoberlinia doka }\end{array}$ & décoction & $\begin{array}{l}\text { petite quantité, } 1 \text { fois /jour, jusqu'à } \\
\text { guérison, per os }\end{array}$ & 2 & 1 \\
\hline
\end{tabular}




\begin{tabular}{|c|c|c|c|c|c|}
\hline & $\begin{array}{l}\text { écorces de Vitellaria paradoxa, de } \\
\text { Khaya senegalensis et de } \\
\text { Pseudocedrela kotschyi + racines } \\
\text { de Parkia biglobosa, de } \\
\text { Piliostigma thonningii et d'Annona } \\
\text { senegalensis + sel rouge }\end{array}$ & décoction & $\begin{array}{l}1 / 2 \mathrm{~L} \text { par sujet/ jour, pendant } 3 \\
\text { jours, per os }\end{array}$ & 7 & 3 \\
\hline & $\begin{array}{l}\text { écorce de Mangifera indica }+ \\
\text { sucre }\end{array}$ & décoction & $\begin{array}{l}\text { 1L adultes et } 1 / 2 \mathrm{~L} \text { jeunes, } 2 \text { fois } \\
\text { /jour, pendant } 5 \text { jours, per os }\end{array}$ & 13 & 6 \\
\hline & $\begin{array}{l}\text { écorces de Parkia biglobosa et de } \\
\text { Strychnos spinosa + potasse }\end{array}$ & décoction & $\begin{array}{l}1 \mathrm{~L} \text { adultes et } 1 / 2 \mathrm{~L} \text { jeunes, } 1 \text { fois / } \\
\text { jour, pendant une semaine, per os }\end{array}$ & 3 & 1 \\
\hline & $\begin{array}{l}\text { écorces de Khaya senegalensis et } \\
\text { de Pterocarpus erinaceus }+ \text { sucre }\end{array}$ & décoction & $\begin{array}{l}\text { 1L adultes et } 1 / 2 \mathrm{~L} \text { jeunes, } 2 \text { fois } \\
\text { /jour, pendant } 5 \text { jours, per os }\end{array}$ & 3 & 1 \\
\hline & écorce d'Adansonia digitata & $\begin{array}{l}\text { poudre }+ \\
\text { eau }\end{array}$ & $\begin{array}{l}\text { 1L adultes et } 1 / 2 \mathrm{~L} \text { jeunes, } 2 \text { fois } \\
\text { /jour, pendant } 5 \text { jours, per os }\end{array}$ & 2 & 1 \\
\hline & $\begin{array}{l}\text { écorces de Mangifera indica et de } \\
\text { Piliostigma thonningii }\end{array}$ & décoction & $\begin{array}{l}\text { 1L adultes et } 1 / 2 \mathrm{~L} \text { jeunes, } 2 \text { fois } \\
\text { /jour, pendant } 3 \text { jours, per os }\end{array}$ & 3 & 1 \\
\hline & $\begin{array}{l}\text { feuilles de Vitellaria paradoxa }+ \\
\text { sucre }\end{array}$ & Pilage & 1L / jour jusqu'à guérison, per os & 2 & 1 \\
\hline & $\begin{array}{l}\text { écorces de Cassia sieberiana et } \\
\text { d'Aganope stulhmannii }\end{array}$ & décoction & $\begin{array}{l}1 \mathrm{~L} \text { adultes et } 1 / 2 \mathrm{~L} \text { jeunes, } 1 \text { fois / } \\
\text { jour jusqu'à guérison, per os }\end{array}$ & 9 & 3 \\
\hline & $\begin{array}{l}\text { écorces de Mangifera indica et de } \\
\text { Pericopsis laxiflora }+ \text { sucre }\end{array}$ & macération & $\begin{array}{l}\text { à volonté, } 2 \text { fois / jour jusqu'à } \\
\text { guérison, per os }\end{array}$ & 11 & 5 \\
\hline & écorce d'Afzelia africana + alcool & $\begin{array}{l}\text { pilage }+ \text { eau, } \\
\text { filtrat }+ \\
\text { alcool }\end{array}$ & $\begin{array}{l}\text { petite quantité, } 2 \text { fois / jour pendant } \\
2 \text { jours, per os }\end{array}$ & 2 & 1 \\
\hline & $\begin{array}{l}\text { écorces de Khaya senegalensis et } \\
\text { de Pseudocedrela kotschyi }\end{array}$ & décoction & $\begin{array}{l}1 \mathrm{~L} \text { adultes, } 1 / 2 \mathrm{~L} \text { jeunes, } 1 \text { fois / } \\
\text { jour, pendant une semaine, per os }\end{array}$ & 3 & 1 \\
\hline & $\begin{array}{l}\text { racines de Sarcocephalus } \\
\text { latifolius, d'Acacia polyacantha, de } \\
\text { Pericopsis laxiflora et de } \\
\text { Strychnos spinosa+ Allium cepa }+ \\
\text { Peper guineense }\end{array}$ & $\begin{array}{l}\text { macération } \\
\text { pendant } 3 \\
\text { jours }\end{array}$ & $\begin{array}{l}\text { à volonté, à volonté, } 1 \text { fois / jour } \\
\text { jusqu'à guérison, per os }\end{array}$ & 2 & 1 \\
\hline & $\begin{array}{l}\text { écorces de Bombax costatum, de } \\
\text { Vitellaria paradoxa et de Detarium } \\
\text { microcarpum }+1 \mathrm{~kg} \text { de sucre }\end{array}$ & décoction & $\begin{array}{l}2 \text { verres bambou/ sujet à jeun, } \\
1 \text { fois / jour, pendant } 3 \text { jours, } \\
\text { per os }\end{array}$ & 2 & 1 \\
\hline & $\begin{array}{l}\text { écorces de Khaya senegalensis et } \\
\text { d'Acacia senegal }\end{array}$ & macération & $\begin{array}{l}1 \mathrm{~L} \text { adultes et } 1 / 2 \mathrm{~L} \text { jeunes, } 1 \text { fois/ } \\
\text { jour, pendant } 3 \text { jours, per os }\end{array}$ & 3 & 1 \\
\hline Piroplasmose & $\begin{array}{l}\text { écorces de Bombax costatum et de } \\
\text { Prosopis africana }\end{array}$ & macération & $\begin{array}{l}2 \text { à } 3 \text { louches, } 1 \text { fois / jour, pendant } \\
5 \text { jours }\end{array}$ & 100 & 1 \\
\hline PPCB & $\begin{array}{l}\text { fruits de Tamarindus indica }+ \\
\text { feuilles de Khaya senegalensis et } \\
\text { de Oxythenanthera abyssinica }\end{array}$ & décoction & $\begin{array}{l}1 / 2 \mathrm{~L} \text { par sujet, } 1 \text { à } 2 \text { fois/ jour, } \\
1 \text { à } 2 \text { jours, per os }\end{array}$ & 100 & 1 \\
\hline
\end{tabular}


H. G. DASSOU et al. / Int. J. Biol. Chem. Sci. 8(1): 189-210, 2014

\begin{tabular}{|c|c|c|c|c|c|}
\hline Plaies & feuilles de Pterocarpus erinaceus & poudre & $\begin{array}{l}\text { à la taille de la plaie, } 3 \text { fois / jour, } \\
\text { pendant } 5 \text { jours, per os }\end{array}$ & 100 & 1 \\
\hline $\begin{array}{l}\text { Rétention } \\
\text { placentaire }\end{array}$ & feuilles de Carica papaya & décoction & $\begin{array}{l}1 \mathrm{~L}, 3 \text { fois / jour jusqu'à guérison, } \\
\text { per os }\end{array}$ & 100 & 1 \\
\hline \multirow[t]{5}{*}{$\begin{array}{l}\text { Tétanie } \\
\text { d'herbage }\end{array}$} & écorce de Pseudocedrela kotschyi & décoction & $\begin{array}{l}2 \mathrm{~L} \text { adultes et } 1 \mathrm{~L} \text { jeunes, } 1 \text { fois / } \\
\text { jour, pendant } 3 \text { jours, per os }\end{array}$ & 18 & 1 \\
\hline & écorce de Detarium microcarpum & pilage + eau & $\begin{array}{l}\text { à volonté, } 1 \text { fois / jour, } \\
\text { pendant } 3 \text { jours, per os }\end{array}$ & 16 & 1 \\
\hline & $\begin{array}{l}\text { écorce de Trichilia emetica }+ \\
\text { fientes de canard }\end{array}$ & poudre & $\begin{array}{l}\text { quelques pincées, } 2 \text { fois en } 1 \text { jour, } \\
\text { per os }\end{array}$ & 16 & 1 \\
\hline & racines de Parkinsonia aculeata & Pilage + eau & $1 \mathrm{~L}$ en jour, per os & 34 & 2 \\
\hline & fruit (pulpe) de Parkia biglobosa & $\begin{array}{l}\text { poudre }+ \\
\text { eau }\end{array}$ & $\begin{array}{l}2 \mathrm{~L} \text { adultes et } 1 \mathrm{~L} \text { jeunes, } 1 \text { fois / } \\
\text { jour, pendant } 2 \text { jours, per os }\end{array}$ & 16 & 2 \\
\hline Toux & écorce de Vitellaria paradoxa & $\begin{array}{l}\text { séchage et } \\
\text { poudre }\end{array}$ & $\begin{array}{l}\text { 1/2 L, } 2 \text { fois /jour, jusqu'à guérison, } \\
\text { per os }\end{array}$ & 100 & 2 \\
\hline \multirow{6}{*}{ Trypanosomose } & $\begin{array}{l}\text { écorce de Kigelia africana }+ \\
\text { chaume de Zea mays }\end{array}$ & $\begin{array}{l}\text { calcination } \\
\text { de chaume } \\
\text { et } \\
\text { macération } \\
\text { du complet }\end{array}$ & $\begin{array}{l}1 \mathrm{~L} \text { adultes et } 1 / 2 \mathrm{~L} \text { jeunes, } \\
2 \text { fois / jour, pendant une semaine, } \\
\text { per os }\end{array}$ & 20 & 3 \\
\hline & $\begin{array}{l}\text { écorce de Khaya senegalensis + } \\
\text { potasse }\end{array}$ & poudre & $\begin{array}{l}\text { à volonté, } 2 \text { fois / jour, } \\
\text { pendant } 3 \text { jours, per os }\end{array}$ & 40 & 7 \\
\hline & $\begin{array}{l}\text { écorce de Khaya senegalensis }+ \\
\text { feuilles de Guieria senegalensis }\end{array}$ & décoction & $\begin{array}{l}2 \mathrm{~L} \text { adultes et } 1 \mathrm{~L} \text { jeunes, } 1 \text { fois / } \\
\text { jour, jusqu'à guérison, per os }\end{array}$ & 20 & 3 \\
\hline & $\begin{array}{l}\text { fruits et feuilles de Crossopteryx } \\
\text { febrifuga }+ \text { sel }\end{array}$ & pilage & $\begin{array}{l}\text { à volonté, } 1 \text { fois / jour, jusqu'à } \\
\text { guérison, per os }\end{array}$ & 6 & 2 \\
\hline & $\begin{array}{l}\text { écorces de Cassia sieberiana, de } \\
\text { Prosopis africana et de Khaya } \\
\text { senegalensis + chaume de } \\
\text { Sorghum bicolor + sel }\end{array}$ & poudre & $\begin{array}{l}\text { petite quantité, } 1 \text { fois / jour, } \\
\text { pendant une semaine, per os }\end{array}$ & 6 & 1 \\
\hline & $\begin{array}{l}\text { feuilles et écorces d'Afzelia } \\
\text { africana et de Combretum } \\
\text { collinum }\end{array}$ & décoction & $\begin{array}{l}2 \text { à } 3 \text { louches, } 1 \text { fois / jour, } \\
2 \text { à } 3 \text { jours, per os }\end{array}$ & 8 & 1 \\
\hline \multirow{2}{*}{ Tuberculose } & $\begin{array}{l}\text { feuilles de Guiera senegalensis }+ \\
\text { sel }\end{array}$ & pilage & $\begin{array}{l}\text { à volonté, } 2 \text { fois /jour, } \\
\text { jusqu'à guérison, per os }\end{array}$ & 20 & 1 \\
\hline & $\begin{array}{l}\text { feuilles de Mitragyna inermis et } \\
\text { d'Anogeissus leiocarpa }\end{array}$ & décoction & $\begin{array}{l}2 \mathrm{~L} \text { adultes et } 1 \mathrm{~L} \text { jeunes, } \\
2 \text { fois /jour, jusqu'à guérison }\end{array}$ & 80 & 5 \\
\hline Variole & $\begin{array}{l}\text { écorce de Pteleopsis suberosa }+ \\
\text { pierre noire }\end{array}$ & macération & à volonté, jusqu'à guérison, per os & 100 & 1 \\
\hline
\end{tabular}


Tableau 5 : FCI et diversité spécifique par catégorie de maladies.

\begin{tabular}{lcc}
\hline Catégories de maladies & FCI & Diversité spécifique \\
\hline Affections gastro-intestinales & 0,5 & 23 \\
Affections cutanées & 0,4 & 16 \\
Affections respiratoires & 0,5 & 31 \\
Affections sanguines & 0,4 & 18 \\
Affections obstétricales (de la reproduction) et autre & 0,5 & 14 \\
\hline
\end{tabular}

FCI : Facteur de Consensus de l'Informateur.

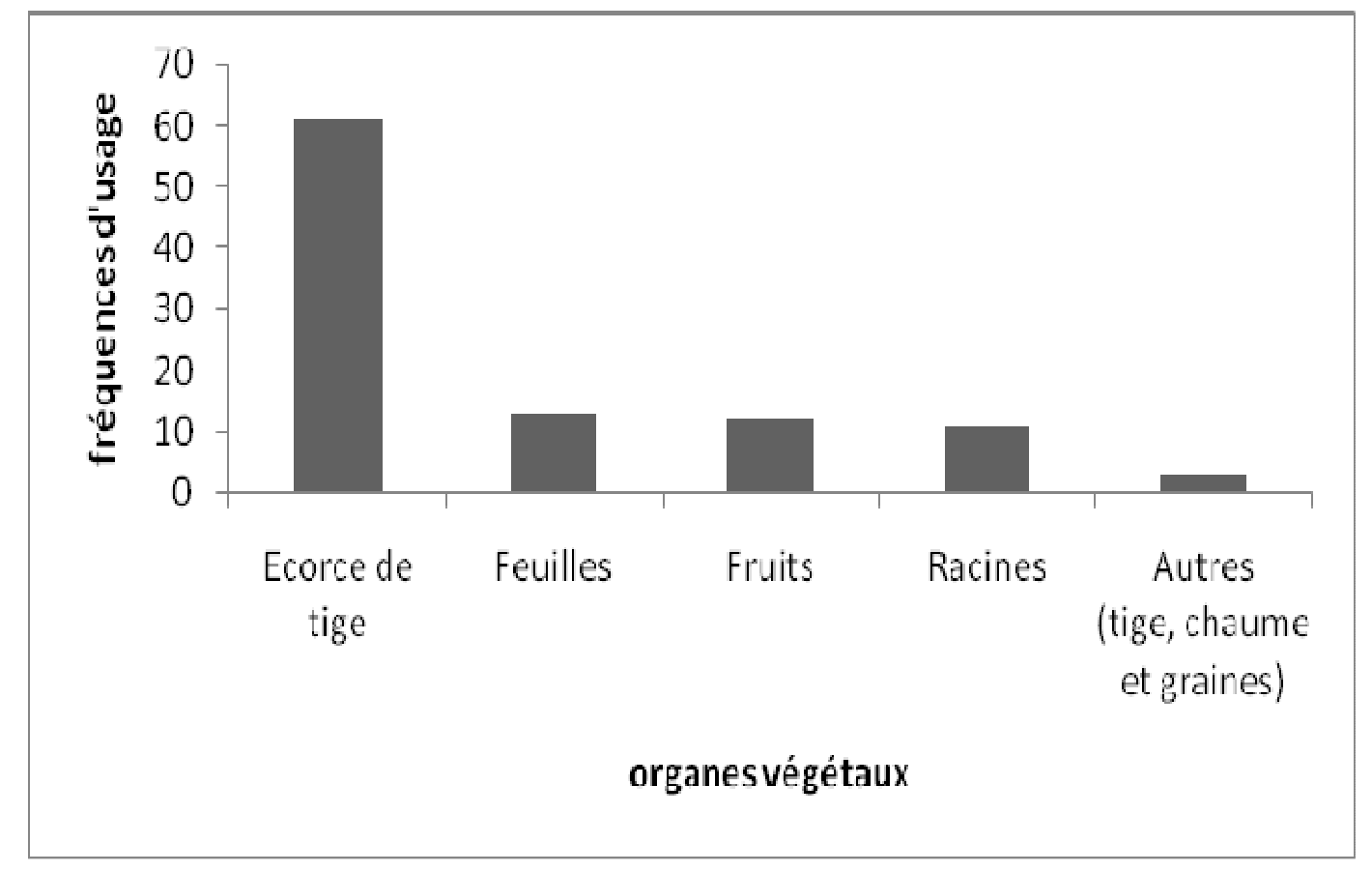

Figure 2: Fréquences d'utilisation des organes végétaux. 


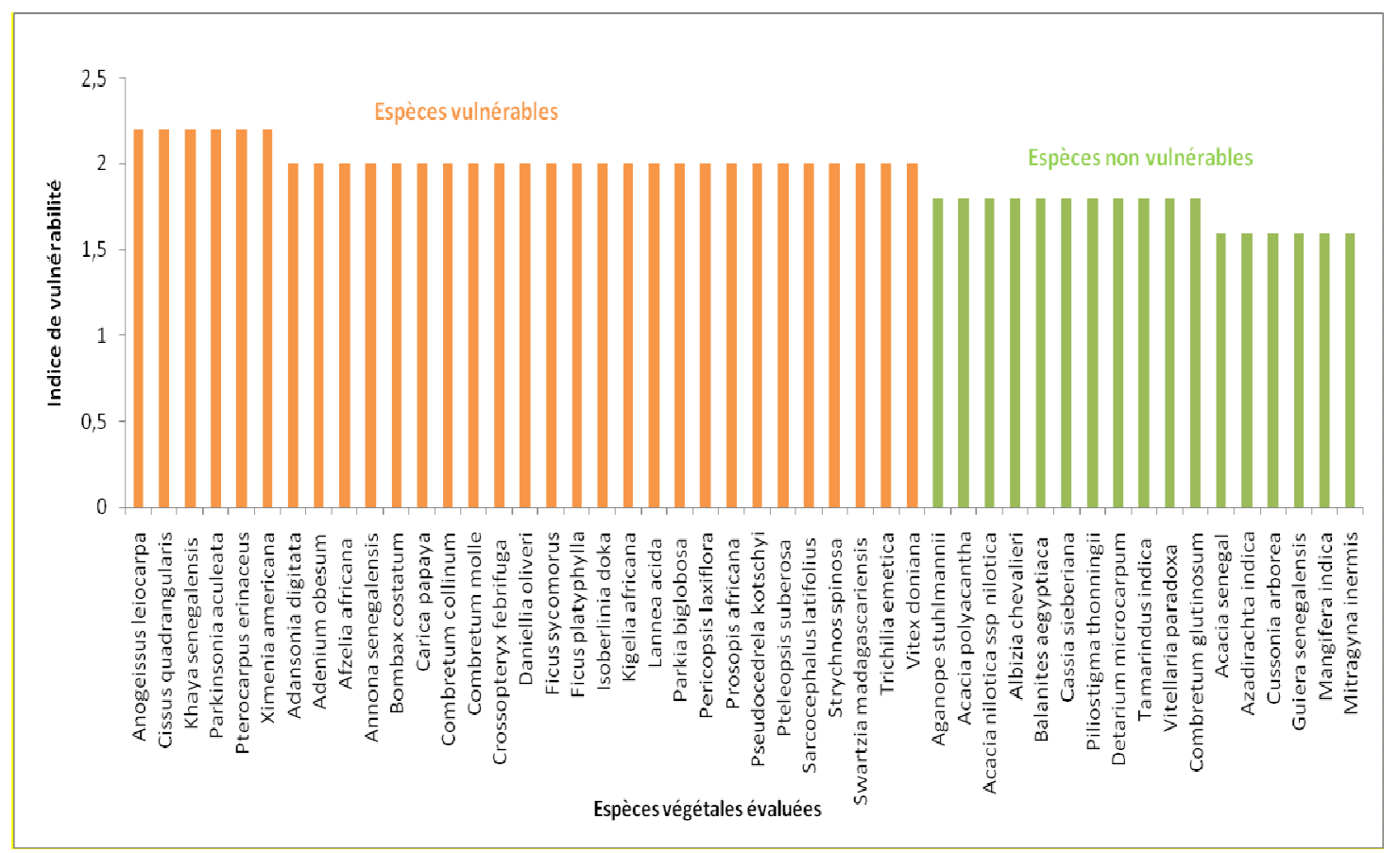

Figure 3: Spectre de vulnérabilité des espèces.

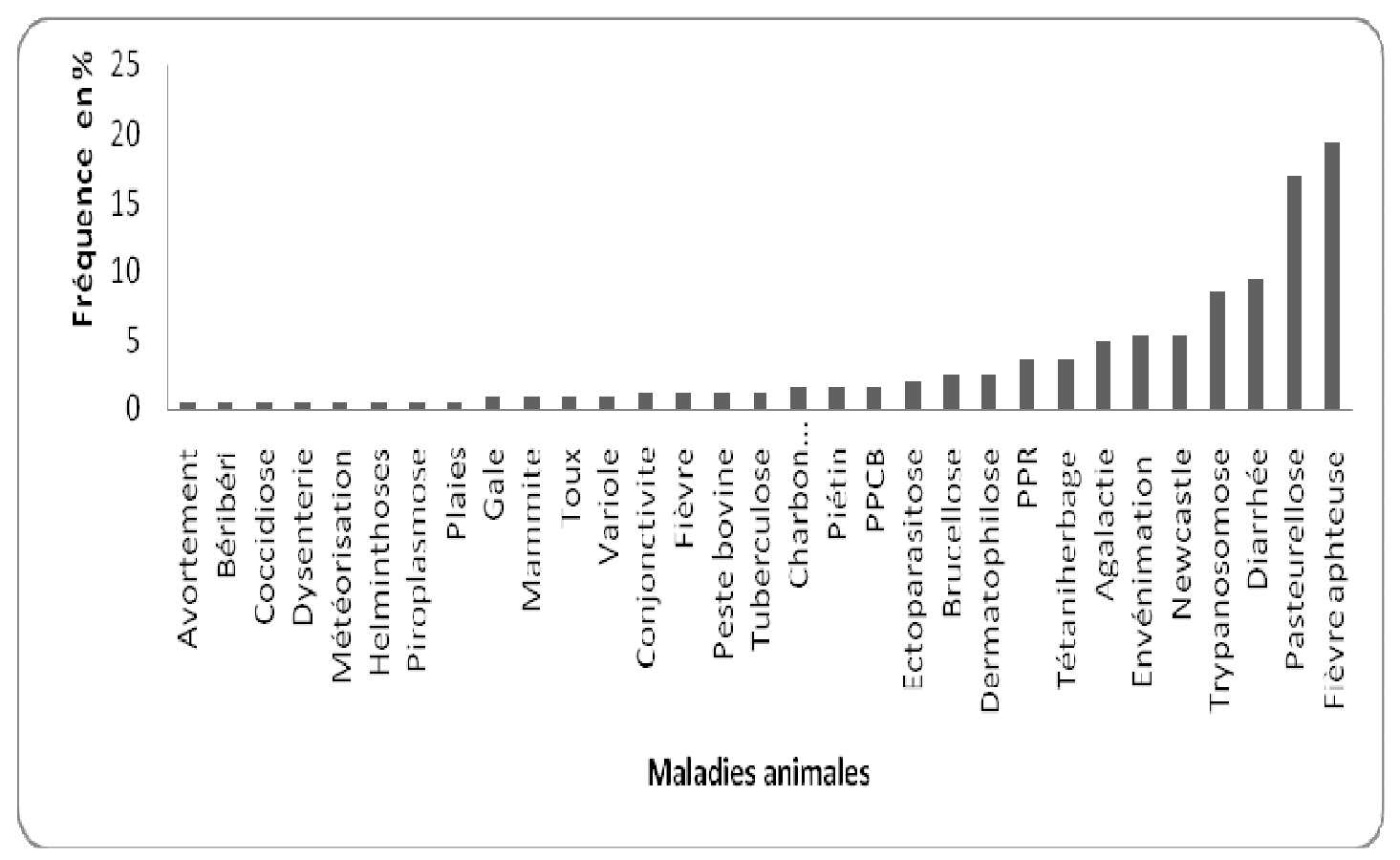

Figure 4: Pathologies animales recensées. 


\section{DISCUSSION}

\section{Savoir ethnovétérinaire}

Le présent travail indiquant une absence de corrélation entre l'âge et le nombre de recettes rapportées et utilisées par chaque enquêté est contraire à d'autres travaux (Upadhyay et al., 2011) ou discussions qui ont porté sur cet aspect. Il a été surtout admis qu'en Afrique, ce sont les sages, c'est-à-dire les personnes les plus âgées, qui détiennent la connaissance traditionnelle de traitement des maladies. L'absence de lien a montré cette étude peut être liée à l'âge moyen de la population qui montre une population relativement jeune. Elle peut aussi s'expliquer par le fait que c'est souvent tout le potentiel ethnovétérinaire qui est transmis des ascendants (sages) aux descendants (jeunes) à telle enseigne que les deux types d'individus ont pratiquement le même niveau de connaissance. Ceci est d'autant plus justifiable car la majorité des personnes enquêtées ont signalé avoir hérité ce savoir de la famille. C'est à juste titre que Tamboura et al. (1998) ont souligné que l'ethnomédecine vétérinaire est une science qui se pratique et se transmet au sein de la société et leur contenu demeure un patrimoine familial.

Flore à usages vétérinaires et affections animales dans la zone cotonnière du Nord-Bénin

Les 56 espèces végétales à usages vétérinaires recensées représentent $02 \%$ de la flore totale du Bénin estimée de 2807 espèces (Akoègninou et al., 2006). Cette richesse spécifique est faible par rapport aux 75 espèces végétales recensées par HounzangbéAdoté (2001) au Sud-Bénin mais les espèces plus fréquentes rapportées par cette étude sont incluses dans celles répertoriées dans la zone cotonnière du Nord-Bénin. En outre, Attindéhou (2012) a recensé 22 espèces de plantes à usages antiparasitaires chez les ruminants au Sud-Bénin. Cette différence de richesse spécifique est due à la diversité spécifique variant d'une zone ou district phytogéographique à un autre à l'intérieur du pays (Adomou, 2011).
Les familles à usages vétérinaires les plus diversifiées en espèces sont les Leguminosae (34\%) et Combretaceae (11\%). L'importance de ces Leguminosae n'est pas une particularité pour les plantes à usages vétérinaires mais pour la flore du Bénin. Parmi les Combretaceae recensées, on note une abondance du genre Combretum, soit $50 \%$ des espèces. Ce qui se comprend car Akoègninou (2006) et Adomou (2011) ont montré la richesse de ce genre dans les savanes arborées et arbustives du district Borgou-Nord auquel appartient la zone d'étude.

Parmi les plantes, Khaya senegalensis est très utilisée. Elle a contribué à $21 \%$ à la constitution des 86 recettes recensées. Elle traite $23 \%$ des maladies et est très sollicitée dans le traitement des helminthoses. Ce qui confirme les travaux de Arbonnier (2009) qui ont aussi montré ses pouvoirs antidiarrhéique et antihelminthique.

Pterocarpus erinaceus et Vitellaria paradoxa sont aussi moyennement sollicitées et entrent chacune dans le traitement de $13 \%$ des maladies citées. Cette contribution de la part de Pterocarpus erinaceus montre sa grande utilisation en médecine traditionnelle. Selon Akoègninou et al. (2006), outre cet usage, elle est fortement demandée dans d'autres domaines comme l'alimentation (le fourrage) et la construction (utilisation comme bois). En ce qui concerne Vitellaria paradoxa, son importance réside dans sa capacité à produire des amandes comestibles et utilisées comme beurre de karité. En effet, ce beurre très apprécié dans la cuisine, est fréquemment utilisé comme onguent dans la fabrication des pommades et cataplasmes. C'est un émollient et un cicatrisant (Arbonnier, 2009). Ce qui justifie son intervention dans la constitution des recettes utilisées pour le traitement de la fièvre aphteuse.

Une plante ayant une contribution faible dans la constitution des recettes, est Balanites aegyptiaca. Elle est plus utilisée pour traiter la fièvre aphteuse. Ce qui corrobore les travaux de (SIFOR, 2009) 
montrant les propriétés cicatrisantes de cette plante sur les plaies animales au Mali.

La méthode de calcul de fréquences a été adoptée dans cette étude pour sélectionner les recettes crédibles. En se référant à la valeur moyenne (0.4) du FCI, on note globalement un consensus faible par rapport à l'usage des phytoremèdes dans la population enquêtée. Considérant les affections respiratoires, le FCI est 0,5 correspondant à une valeur moyenne et témoigne d'un consensus moyen dans l'utilisation de quelques plantes pour traiter cette catégorie de maladies. Dans ce sens, on comprend la sélection de Anogeissus leiocarpa associée à Mitragyna inermis qui sont très indiquées dans le traitement de la tuberculose. Ceci est intéressant car les écorces de racine de Anogeissus leiocarpa ont été déjà citées comme antitussives (SIFOR, 2009). Cette même plante possède d'excellentes propriétés antihelmintiques. Ce pouvoir a été déjà révélé par Kaboré (2009) à partir de tests in vitro et in vivo sur les strongles gastrointestinaux des ovins de race mossi du Burkina Faso.

Pseudocedrela kotschyi + Khaya senegalensis constituent la composition de plantes sélectionnées pour traiter la maladie de Newcastle. La potentialité de Khaya senegalensis dans le traitement des pathologies n'est plus à démontrer. De l'autre côté, Adeniyi et al. (2010) et Ayo et al. (2010) ont rapporté l'activité antimicrobienne de Pseudocedrela kotschyi, les feuilles pour les premiers et le tronc pour les seconds.

La valeur 0,5 obtenue pour les affections obstétricales et gastrointestinales est aussi moyenne. Dans le premier cas, elle permet de supposer que les éleveurs s'intéressent aux maladies qui limitent la productivité animale pour éviter les grandes pertes économiques liées aux avortements et mortalité des animaux. Dans le second cas, ce niveau de connaissance de plantes peut être lié au fait que les pathologies gastrointestinales sont fréquentes dans la plupart des élevages et surtout chez l'homme où la plupart des plantes proposées sont utilisées.
Les 31 maladies animales recensées dans la zone d'étude sévissent dans beaucoup de troupeaux en Afrique de l'Ouest. Elles font parties des maladies décrites par MCF (1998) et sont souvent dues à des parasites ou des bactéries, etc.

Tous les organes végétaux importants sont impliqués dans la préparation des médicaments traditionnels à usages vétérinaires. Il s'agit des écorces de tige, feuilles, fruits et racines. L'usage majoritaire de ces organes pour la constitution des recettes a été déjà signalé par beaucoup d'autres auteurs (Deleké, 2005; Fagnissè, 2006). L'intérêt pour ces organes est dû à leur importance dans la physiologie de la plante. En effet, les racines constituent l'organe d'absorption de l'eau et des éléments minéraux et par ricochet lieu de constitution de la sève brute; ensuite les tissus du bois sont les conducteurs des sèves brute et élaborée, puis les fruits sont les accumulateurs de réserves importantes.

\section{Vulnérabilité des espèces}

Le fort taux d'espèces vulnérables obtenu dans cette étude montre la pression élevée exercée sur ces ressources. La vulnérabilité d'une plante dépend en grande partie de son type morphologique, de sa fréquence dans le milieu, des usages qu'on en fait et surtout de ses organes sollicités (Cunningham, 1996).

En tenant compte du type morphologique, la majorité des espèces recensées sont des arbres. Ainsi, à cause de leur port dressé, outre leur utilisation en médecine vétérinaire, ils sont très utilisés comme bois de construction. C'est surtout le cas Pterocarpus erinaceus, Prosopis africana, Afzelia africana... dont l'abattement systématique est à l'origine de la réduction de leur population.

Aucune nécessité d'économie liée à une ressource n'est souvent notée dans un milieu si la ressource est abondante (disponible) même en cas non renouvellement de celle-ci. En effet, les populations en abusent et procèdent souvent à des récoltes 
incontrôlées. C'est le cas de Vitellaria paradoxa et de Balanites aegyptiaca, très fréquentes dans la zone d'étude et qui font l'objet de massacre par la population. Heureusement, Vitellaria paradoxa fait partie des plantes non vulnérables. Cette absence de vulnérabilité peut s'expliquer par le fait qu'elle soit protégée par les agrosystèmes traditionnels installés par la population. Notons que les espèces moins fréquentes sont plus sujettes à la vulnérabilité. Curieusement, ce sont des espèces très utilisées mais sont souvent détruites à la recherche de terres pour la culture du coton et autres.

La majorité des plantes recensées par ce travail sont utilisées à d'autres fins. C'est le cas de Pteleopsis suberosa très utilisée pour la fabrication de corde dans l'artisanat, de Khaya senegalensis dans le traitement des affections chez l'homme, de Pterocarpus erinaceus dans le fourrage chez les bovins...Ce qui confirme leur vulnérabilité.

Les fruits, les graines, les écorces de tige et les racines sont les organes dont l'utilisation abusive a des impacts écologiques graves sur les espèces. En ce qui concerne les fruits, on peut envisager un impact sur le processus de régénération car selon Deleke (2005) le maintien de la capacité de régénération dépend entre autres de la disponibilité des semences. L'écorçage permanent est souvent responsable des troubles physiologiques influençant forcément la fructification et par conséquent la formation des graines assurant la pérennité de l'espèce (Traoré et al., 2011). Les racines étant le siège de la nutrition minérale et en eau d'une plante, son utilisation abusive est en général fatale pour la plante surtout que $93 \%$ des espèces ne sont pas cultivées pour assurer la disponibilité des ressources.

Parmi les 6 espèces plus vulnérables distinguées dans cette étude, se trouvent Khaya senegalensis, Kigelia africana et Pterocarpus erinaceus. Ce qui corrobore les travaux de Adomou et al. (2011) qui ont été déjà signalées comme plantes menacées au Bénin.

\section{Conclusion}

La flore à usages vétérinaires recensée constitue $02 \%$ de la flore totale du Bénin. Parmi elle, Khaya senegalensis, Cassia sieberiana, Pterocarpus erinaceus, Vitellaria paradoxa sont les plantes les plus citées et sont utilisées pour traiter les plus grands nombres de maladies. La majorité des plantes citées sont disponibles dans la zone d'étude et les populations arrivent à les récolter pour les divers usages.

Les maladies les plus fréquentes affectant les animaux dans la zone d'étude sont la Fièvre aphteuse et la Pasteurellose. Elles sont traitées par beaucoup de plantes dont les plus crédibles sélectionnées dans ce travail sont: Balanites aegyptiaca et Acacia nilotica ssp. nilotica pour la Fièvre aphteuse ; Mangifera indica seule ou associée à Khaya senegalensis et Cassia sieberiana pour la Pasteurellose. Les tests in vitro et in vivo sur les animaux et les recherches phytochimiques ultérieures sont nécessaires pour la valorisation des potentialités des plantes citées dans le traitement des pathologies animales rencontrées dans les élevages au Bénin.

$\mathrm{Au}$ total, les populations, en plus des interventions des vétérinaires modernes dans leur élevage, n'ont pas abandonné l'ethnomédecine vétérinaire. Elle constitue un appoint surtout en cas de manque de moyens financiers.

\section{REMERCIEMENTS}

Nous remercions très sincèrement l'Université d'Abomey-Calavi pour le financement de ces travaux par le biais du Programme Fonds Compétitifs de Recherche (PFCR/UAC, $2^{\text {ème }}$ phase). Nous exprimons toute notre gratitude à tous les membres de l'équipe du Projet PHARMAVET et particulièrement du Laboratoire de Botanique et Ecologie Végétale de la Faculté des Sciences et Techniques dirigé par le Professeur AKOEGNINOU Akpovi. Nos remerciements vont également à l'endroit des populations locales, des Responsables du Développement Rural (RDR) et des Conseillés en Production Animale qui ont 
énormément contribué au bon déroulement des enquêtes de terrain.

\section{REFERENCES}

Adeniyi CBA, Odumosu BT, Aiyelaagbe OO and Kolude B. 2010. In vitro Antimicrobial activities of methanol extracts of Zanthoxylum zanthoxyloides and Pseudocedrela kotschyi. African J. Biomed. Res., 13: 61-68.

Adomou AC, Agbani OP, Sinsin B. 2011. Plantes. In Protection de la Nature en Afrique de l'Ouest: Une Liste Rouge pour le Bénin. Nature Conservation in West Africa: Red List for Benin, Neuenschwander P, Sinsin B, Goergen $\mathrm{G}$ (eds). International Institute of Tropical Agriculture: Ibadan, Nigeria; 21-46.

Akoègninou $\mathrm{A}$, van der Burg $\mathrm{WJ}$, van der Maesen LJG. 2006. Flore Analytique du Bénin. Backhuys Publishers: Wageningen; 1034p.

Akoègninou A. 2004. Recherches botaniques et écologiques sur les forêts actuelles du Bénin. Thèse d'Etat. Université de Cocody-Abidjan (Côte d'Ivoire). 326p.

Andrade-Cetto A, Heinrich M. 2011. From the field into the lab: useful approaches to selecting species based on the local knowledge. fphar., 2: 1-3.

Arbonnier M. 2009. Arbres, Arbustes et Lianes des Zones Sèches d'Afrique de l'Ouest ( $3^{\mathrm{e}}$ edn). Ed. Quae, MNHN. Imp. Louis Jean Imprimeur: Paris ; 355.

Attindéhou S, Houngnimassoun MA, Salifou S, Biaou CF. 2012. Inventorying of herbal remedies used to control small ruminant's parasites in Southern Benin. International Multidisciplinary Research Journal, 2(8):14-16.

Ayo RG, Audu OT, Ndukwe GI, Ogunshola AM. 2010. Antimicrobial activity of extracts of leaves of Pseudocedrela kotschyi. African J. Biotech., 9(45): 7733-7737.

Betti JL. 2001. Vulnérabilité des plantes utilisées comme antipaludiques dans l'arrondissement de Mintom au sud de la réserve de Biosphère du Dja (Cameroun). Syst. Geogr. Pl., 71: 661678.

Cunningham AB. 1996. Peuples, Parc et Plantes. Recommandation pour les zones à usages multiples et les alternatives de développement autour du parc national de Bwindi impénétrable (Ouganda). Document de travail Peuples et Plantes $\mathrm{n}^{\circ}$ 4. UNESCO, Paris ; p.64.

DE. 2010. Annuaire statistique sur l'élevage. Direction de l'Elevage, pp. 09-82.

Deleke I. 2005. Utilisation des plantes médicinales contre les maladies et troubles gynécologiques dans les terroirs autour de la zone cynégétique de la Pendjari du Bénin: compréhension, inventaire et perspective pour leur conservation. Mémoire du Diplôme d'ingénieur agronome. FSA/UAC. Bénin. 70p.

Devendrakumar D, Anbazhagan M. 2012. Ethnoveterinary medicinal plants used in Perambalur District, Tamil Nadu. Research in Plant Biology, 2 (3): 24-30.

Hounzangbé-Adoté SM. 2001. L'élevage face à la pharmacopée en médecine vétérinaire au sud du. Bénin. Bulletin de la Recherche Agronomique, 33 : 1-9.

Fagnissè F. 2006. Valorisation des plantes médicinales dans le traitement des maladies des ruminants (cas des bovins) aux alentours du parc $\mathrm{W}$ : inventaire ethnobotanique et perspectives. Mémoire de DIT/EPAC/UAC. 114p.

FAOSTAT. 2010. FAO Statistics Division, http://faostat.fao.org.

INSAE. 2013. Recensement Général de la Population et de l'Habitat (Résultats Provisoires). MDAEP. INSAE : Cotonou, Bénin ; 8p.

Kaboré A. 2009. Activité anthelminthique de deux plantes tropicales testée in vitro et in vivo sur les strongles gastrointestinaux des ovins de race mossi du Burkina Faso. Thèse de Doctorat. Université Polytechnique de BoboDioulasso. 167p. 
MCF (Ministère de la Coopération Française). 1998. Hygiène et Pathologie. In Mémento de l'Agronome ( $4^{\mathrm{e}}$ edn). Imprimerie CHIRAT : France ; 12091258.

MEPN (Ministère de l'Environnement et de la Protection de la Nature). 2008. Programme d'Action National d'Adaptation aux changements climatiques du Bénin (PANA-BENIN), $81 \mathrm{p}$.

Okafor J, Ham R. 1999. Identification, utilization, and conservation of medicinal plants in southeastern Nigeria. Issues in Africa Biodiversity 3: 1-7.

SIFOR. 2009. Répertoire des espèces forestières ligneuses des régions de Mopti, Tombouctou et Gao. Rapport National, 181 p.

Tamboura H, Kaboré H, Yaméogo SM. 1998. Ethnomédecine vétérinaire et pharmacopée traditionnelle dans le plateau central du Burkina Faso : cas de la province du Passoré. Biotechnol. Agron. Soc. Environ. 2(3) : 181-191.

Traoré L, Ouédraogo I, Ouédraogo A, Thiombiano A. 2011. Perceptions, usages, et vulnérabilité des ressources végétales ligneuses dans le Sud-Ouest du Burkina-Faso. Int. J. Biol. Chem. Sci., 5(1): 258-278.

Ugulu I. 2009. The investigation and quantitative ethnobotanical evaluation of medicinal plants used around Izmir province, Turkey. Journal of Medicinal Plants Research, 3(5): 345-367.

Upadhyay B, Singh KP, Kumar A. 2011. Ethno-veterinary uses and informants consensus factor of medicinal plants of Sariska region, Rajasthan, India. Journal of Ethnopharmacology, 133: 14-25. 